\title{
The impact of the risk of COVID-19 on Black, Asian and Minority Ethnic (BAME) members of the UK dental profession
}

\author{
Chet Trivedy, ${ }^{{ }_{1}}$ Ian Mills ${ }^{2}$ and Onkar Dhanoya ${ }^{3}$
}

\section{Key points}

Reviews the impact of COVID-19 infections for the dental profession.

Highlights potential challenges faced by BAME members of the dental profession in light of the COVID-19 pandemic
Develops a call for action for dental governing bodies and policymakers to urgently address these risks and support the dental profession in delivering high-quality patient care, while safeguarding the health and welfare of the dental team.

\begin{abstract}
There is growing evidence that Black, Asian and Minority Ethnic (BAME) groups in the UK are at increased risk of death from coronavirus (COVID-19), with Black Afro-Caribbean, Indian, Pakistani and Bangladeshi populations being particularly at risk. Although the reasons are unclear, it is likely to be a combination of cultural and socioeconomic, as well as the higher prevalence of co-morbidities such as high blood pressure, cardiovascular disease, raised body mass index (BMI) and type 2 diabetes in these populations. The NHS is advising that frontline staff of BAME origin should be risk-assessed regarding their safety in working in clinical areas which are at risk of COVID-19. There is, however, little evidence on the risks posed to the BAME community within the dental profession. This paper outlines some of the challenges faced, and advocates that urgent action needs to be taken to mitigate the risks of COVID-19 and ensure BAME staff safety upon returning to work.
\end{abstract}

\section{Background}

An article published in the Guardian newspaper on 30 April 2020 highlighted that the NHS was providing specific advice for frontline staff from Black, Asian and Minority Ethnic (BAME) groups to undergo a risk assessment for their fitness to work with regards to the coronavirus (COVID-19) pandemic. ${ }^{1}$ The UK, which has one of the highest rates of COVID-19-related deaths globally, reported 276,322 confirmed cases and 39,045 deaths as of 2 June 2020 . However, the UK has also registered more than 59,000 more deaths then

${ }^{1}$ Consultant in Emergency Medicine, Brighton and Sussex University Hospital, Senior Lecturer in Emergency and Resuscitation Care, Blizard Institute, Queen Mary University of London, UK; ${ }^{2}$ Dean of the Faculty of General Dental Practice (UK), Honorary Associate Professor, Peninsula Dental School, General Dental Practitioner, Torrington Dental Practice, Devon, UK; ${ }^{3}$ General Dental Practitioner, Honour Health, Newcastle, Honorary Clinical Lecturer,

Newcastle Dental School, UK.

${ }^{*}$ Correspondence to: Chet Trivedy

Email address: chetan.trivedy@nhs.net

Refereed Paper.

Accepted 4 June 2020

https://doi.org/10.1038/s41415-020-1781-6 expected since 20 March 2020, suggesting the direct and indirect impact of COVID-19 may be significantly higher. ${ }^{2,3}$

There is a view that BAME groups are at increased risk of death from COVID-19 and there is growing evidence to suggest that Black Afro-Caribbean people and those from the Indian subcontinent (India/Pakistan/ Bangladesh/Sri Lanka) are particularly at risk. A recent rapid data and evidence review from the Centre for Evidence-Based Medicine (CEBM) found that excess hospital deaths due to COVID-19 were 1.5 times higher than expected for Indians living in the UK, 2.8 times higher for the Pakistani population and 3 times higher for the Bangladeshi population. ${ }^{4}$ The Black communities' figures were even higher, with the Black African population having 4.3 times higher hospital deaths than expected. For the Black Caribbean group, it was 2.5 times higher, and for other BAME groups, it was 1.6 times higher. This finding has also been corroborated by the Office for National Statistics (ONS). Figure 1 shows the odds ratio of the chances of dying of COVID-19 for the BAME group compared to the White British population. It is interesting to note that Indian females appear to be at higher risk than their male counterparts, whereas the reverse is true for Bangladeshi/Pakistani and Chinese populations. ${ }^{5}$ Intensive Care National Audit and Research Centre (ICNARC) data have also confirmed increased numbers of COVID19-related admissions to intensive care from BAME groups in critical care units across the UK during the pandemic. ${ }^{6}$ These findings have also been supported by a recent report (COVID19: Review of Disparities in Risks and Outcomes) published by Public Health England (PHE), which stated those of Bangladeshi ethnicity had twice the risk of death from COVID-19 when compared to those of White British ethnicity, and people from other ethnicities (Chinese, Indian, Pakistani, other Asian, Caribbean and other Black ethnicity) had between 10-50\% higher risk of death when compared to White British. $^{7}$ Given that the 2011 UK census estimated that $13 \%$ of the population, which represents 8.65 million people, are from a BAME background, this represents a significant percentage of the population which may be at risk from COVID-19. ${ }^{8}$ 


\section{COVID-19-related deaths in UK healthcare workers}

A disproportionate number of deaths attributed to COVID-19 in BAME healthcare workers (HCWs) has also been reported. The latest figure at the time of writing (2 June 2020) confirmed at least 243 known deaths of health and social care workers since the lockdown began on 25 March 2020. ${ }^{9}$ The analysis of 35 nurses/midwives, 27 HCWs, 19 doctors/dentists and 25 'other staff' (106 in total) published in the Health Service Journal found that, of the staff who died, $71 \%$ nurses and midwives, 56\% HCWs, $94 \%$ doctors and dentists (the actual number of dentists in this group was not specified) and 29\% 'other staff' were from a BAME background. ${ }^{10}$ The data confirms that, even in the health sector, BAME HCWs appear to be at a higher risk of a COVID-19-related death. Although the authors noted that it was not possible to determine if the COVID-19 infection was contracted during work, it stated that all of the subjects were currently working during the pandemic at the time of testing positive for COVID-19. Additionally, although the numbers were small, they felt doctors who died tended to be older males. Several theories have been proposed for the higher BAME-associated deaths, including cultural factors like living in extended families, social inequalities and increased co-morbidities such as high blood pressure, diabetes, cardiovascular disease, renal disease and obesity, all of which are more prevalent in BAME groups. The answers are likely to be multifactorial, but there is now widespread acknowledgement that BAME healthcare workers are at a higher risk of COVID-19-related deaths. ${ }^{11,12}$

\section{Impact of COVID-19 on BAME dental professionals}

NHS data from March 2019 suggest that, of the 1.2 million NHS workers, approximately $20.7 \%$ were from BAME groups. Specifically, in relation to doctors, $45 \%$ of all doctors across the grades were from a BAME background..$^{13}$ In comparison, the British Dental Association (BDA) quote that $28 \%$ of UK dentists are of BAME origin..$^{14}$ It could be that a significant number of doctors and dentists working in the UK are already at increased risk of dying of COVID-19 as a part of the general population and that is even before consideration of any work-related activity in the clinical setting.

Fig. 1 The odds ratio (risk) of death from COVID-19 according to ethnicity (reproduced with permission from the UK Office of National Statistics) ${ }^{5}$

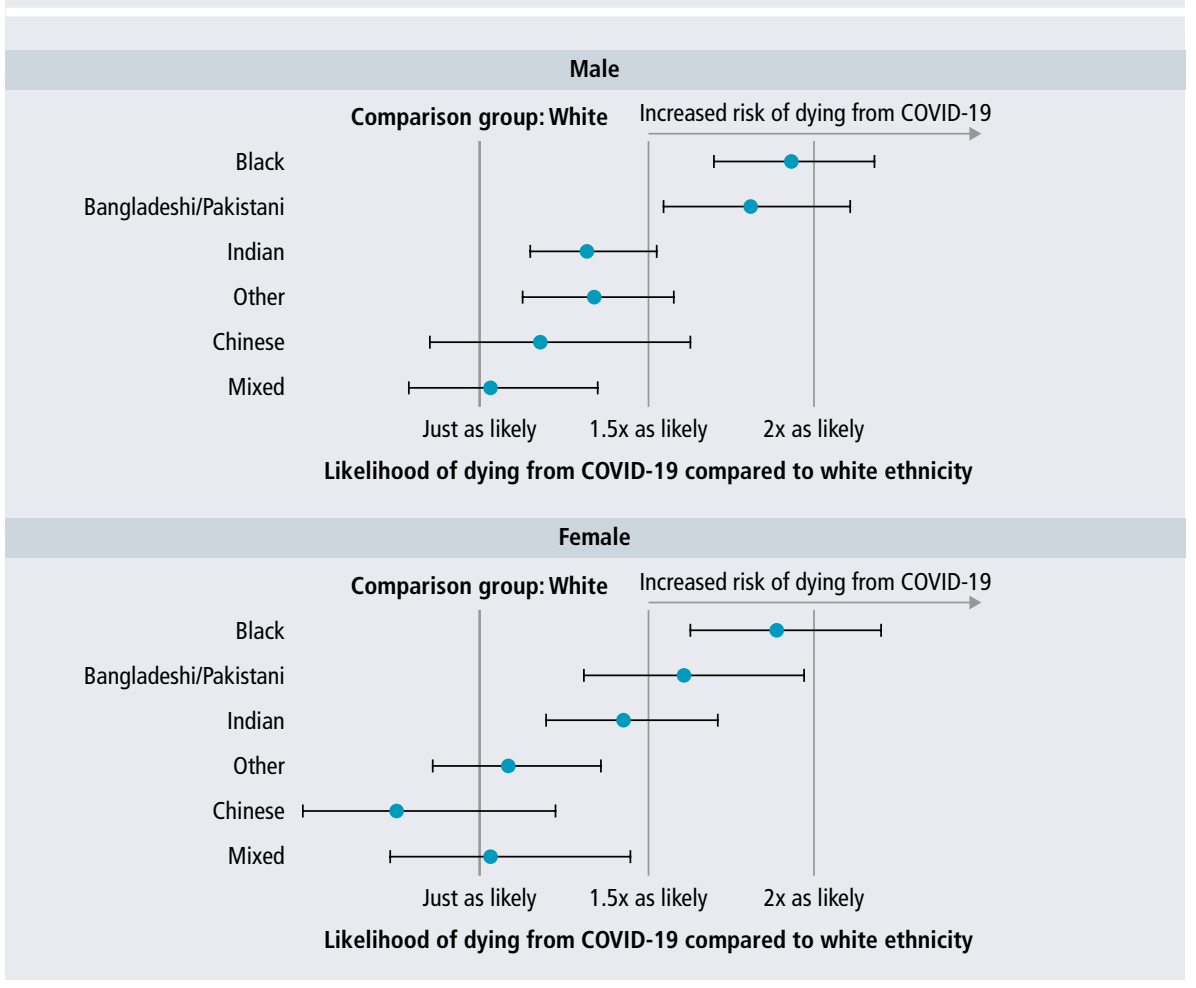

It is therefore critical that the government, including PHE, NHS England and professional bodies, prioritise research in this area and, in the meantime, provide robust guidance and measures focusing on risk stratification to mitigate the risk. One recent proposal has been to risk-assess healthcare workers, who may be at high risk potentially in terms of age, sex, ethnicity and other health considerations..$^{15}$ Although it may be feasible and practical for some members of the medical profession who are deemed to be too high risk to have patient-facing roles and can either be redeployed or work from home, it is a completely different situation for the dental profession. Virtually all aspects of clinical dentistry involve face-to-face close contact work with patients; therefore, working from home or having non-patient-facing roles is not feasible. Additionally, those working in primary care will be self-employed, either performing within a target-remunerated NHS contract or in private practice where fees are sourced from the patient. In either system, the dental practice is a small business with the cost of staffing, materials, consumables, facility costs and mortgages or rent on the premises. Many dentists will have significant borrowing on premises and items of equipment, and such outgoings need to be serviced to keep the business solvent.

It is important to recognise that many dental care professionals (DCPs) will face the same risks as the dentist. Indeed, in terms of the routine work undertaken, dental hygienists may be at greater risk in view of the frequent use of ultrasonic scalers, which may be considered as a significant aerosol generating procedure (AGP). DCPs are likely to be employees and this may pose a challenge for both the employer and the DCP, should the employee be from a BAME group. Consideration will need to be given as to the risk and how this can be minimised within the clinical environment. It would be prudent to perform a risk assessment and seek specialist advice, and there are a number of risk assessment tools which may support the dental team facilitate this process. ${ }^{16,17,18}$

\section{Mitigating AGPs and aerosol generated exposure}

The issue around risk has been further compounded by confusion over guidance and the use of personal protective equipment (PPE) for the dental profession. There is growing concern among many medical professionals that clinical work in the proximity of the aero-digestive tract (mouth/nose/pharynx/ airways) creates a higher risk for COVID-19. The Resuscitation Council UK ${ }^{19}$ and the British Association of Maxillofacial Surgery/ENT UK ${ }^{20}$ suggest that, in addition to AGPs produced 
during dental treatment, practitioners must also consider the impact of aerosol generated exposure (AGE). AGE results from exposure to the aerosol-related secretions that are produced as a result of activities such as coughing, talking, retching and sneezing, by the generation of droplets and aerosols when working in close proximity to the oral and nasal cavity. This is clearly an occupational risk for dental professionals, who often work within 50 centimetres of the oral and nasal cavity. It is therefore important to note that a dental extraction or oral examination, which may not necessarily constitute an AGP, is likely to have some risk from AGE. It remains to be determined how significant this may be, but is supported by a report published by the ONS which suggests that the dental profession has the highest potential occupational exposure to COVID-19 due to close and frequent patient contact involving both AGP- and AGE-related activities. ${ }^{21}$ The Faculty of General Dental Practice (UK) have produced clinical guidance on supporting a return to practice for the primary care dental team. ${ }^{22}$ They support the use of the term AGE and advocate that this is considered in terms of low risk or high risk. A risk assessment is undertaken based on a number of factors, including national alert level, risk of procedure and personal risk. At present, there are no data to show how much extra risk is posed by ethnicity and any co-morbidities in the presence of AGP/AGE risks when compared to the general population. We have to be mindful that, since the lockdown, the routine practice of clinical dentistry in the UK has ceased. It may be some time before dentists return to normal working in sufficient numbers to enable these risks to be measured. However, a recent report found that six dentists have recently died from COVID-19 in Indonesia as well as a dental nurse from the UK. ${ }^{9,23}$ It is important to acknowledge, however, that it is unclear whether transmission was related to occupational risks. Other studies from China and Italy have also confirmed the risks to the dental profession from COVID-19. ${ }^{24,25}$

\section{Potential pitfalls in identifying COVID-19 patients in the dental surgery}

Recent data from the CEBM suggest that between 5-80\% of COVID-19 infections may be asymptomatic, which makes any risk assessments based on key symptoms such as temperature or a cough potentially unreliable. ${ }^{26}$ As a consequence, there is a potential risk that a patient presenting with a dental infection, who may have a low-grade fever, may be denied treatment on the basis of having active COVID-19 symptoms. As a result, we advise that each patient is risk-assessed on a case-by-case basis, taking their medical history, social history and potential exposure to COVID-19 into consideration.

\section{Responding to the concerns of the dental profession}

It is essential that the UK government, regulatory bodies and professional bodies come together and respond to the needs of their membership. This is particularly important for the dental profession, as a majority of them will be independent practitioners with selfemployed status who will not have the option of redeployment or being able to shield until a vaccine or cure becomes available.

\section{The applicability of global guidance to the UK COVID-19 scenario}

Evidence from other countries should be considered, albeit with caution, as other countries have a different rate of exposure to COVID-19. As national policies and guidance will be made in accordance to exposure to COVID-19, care has to be taken when extrapolating guidance from other countries who have a significantly lower population exposure to COVID-19. It is also important to note that each country may be on a different trajectory from the UK in terms of its exposure to COVID-19 and has taken different measures to control the pandemic, making direct comparisons more difficult.

\section{Inequalities for dental professionals}

It is imperative that any guidance is fit for purpose for all members of the dental team, as some will have co-morbidities that will put them at higher risk even if they are not BAME, and guidance should ensure all stakeholders in the dental profession are supported to make a safe and effective transition back to clinical work. Some dental professionals, due to their religious beliefs (for example, Sikh dental professionals who are required to keep their beards), may find it harder to wear certain types of PPE, which may compromise their safety in terms of exposure to COVID-19.
Although solutions such as the use of a powered air-purifying respirator (PARP) are available as an option, the choice of PPE should be considered following a risk assessment.

\section{Geographical variation in COVID-19 infection rates}

The risk to dental professionals will vary according to geographical locations, with London, East of England, Birmingham and the Black Country having some of the highest rates of COVID-19related critical care hospital admissions. ${ }^{5}$ Also, there is no guarantee that we will not be exposed to further waves of COVID-19 as the lockdown rules are relaxed. As a result, dental professionals will need to plan realistic timelines regarding what is feasible and how additional treatments may need to be phased in.

\section{Summary}

The urgency of forward planning cannot be overemphasised, given the short timescale before practices open up for patient care. We have to acknowledge the paucity of highquality evidence to support policy-making and it is vital that this issue is addressed as a matter of urgency. Research funding must be made available and directed towards projects which aim to address the evidence gap that exists within COVID-19 and dentistry. This will not only allow the dental profession to shape the immediate response to the practice of safe dentistry, but also help generate a blueprint for any future pandemics.

Any additional risks to staff from COVID-19, whether that is due to ethnicity, health or risk of exposure to COVID-19, should be carefully assessed and factored in when planning a return to work for the dental profession. The dental team should be provided with the most relevant and appropriate guidance from dedicated occupational health services and resources, such as PPE, as well as the training to facilitate this into clinical practice. The dental profession must be supported in its drive to return to work to provide ongoing oral healthcare which the population desperately needs. However, safety must continue to take priority, and we must ensure that every risk is considered diligently and mitigated wherever possible. This must include the risk related to ethnicity and it is important that there is adequate BAME representation when developing any monitoring or policy development relating to this topic. 


\section{References}

1. Campbell D. NHS looks into taking BAME staff off frontline for their safety. The Guardian (London) 2020 April 29.

2. Worldometer. Coronavirus Cases. 2020. Available online at https://www.worldometers.info/coronavirus/? (accessed May 2020).

3. Burn-Murdoch J, Giles C. UK suffers second-highest death rate from coronavirus. Financial Times (London) 2020 May 28.

4. Razaq A, Harrison D, Karunanithi S, Barr B, Asaria M, Khunti K. BAME COVID-19 Deaths - What do we know? Rapid Data \& Evidence Review: 'Hidden in Plain Sight'. 2020. Available at https://www.cebm.net/wp-content/ uploads/2020/05/BAME-COVID-Rapid-Data-EvidenceReview-Final-Hidden-in-Plain-Sight-compressed.pdf (accessed May 2020).

5. Office for National Statistics. Coronavirus (COVID-19) roundup. 2020. Available at https://www.ons.gov.uk/ peoplepopulationandcommunity/healthandsocial care/conditionsanddiseases/articles/coronavirus covid19roundup/2020-03-26 (accessed May 2020).

6. ICNARC. ICNARC report on COVID-19 in critical care. 2020. Available online at https://www.icnarc.org/OurAudit/Audits/Cmp/Reports (accessed May 2020).

7. Public Health England. COVID-19: review of disparities in risks and outcomes. 2020. Available online at https:// www.gov.uk/government/publications/covid-19-reviewof-disparities-in-risks-and-outcomes (accessed May 2020).

8. UK Government. UK population by ethnicity. Available online at https://www.ethnicityfactsfigures.service.gov. uk/ukpopulationby-ethnicity (accessed June 2020).

9. Nursing Notes. Memorial of Health \& Social Care Workers taken by COVID-19. 2020. Available at https:// nursingnotes.co.uk/covid-19-memorial/ (accessed May 2020).
10. Cook T, Kursumovic E, Simon L. Exclusive: deaths of NHS staff from covid-19 analysed. 2020. Available at https:// www.hsj.co.uk/exclusive-deaths-of-nhs-staff-fromcovid-19-analysed/7027471. article (accessed May 2020).

11. Rimmer A. Covid-19: Disproportionate impact on ethnic minority healthcare workers will be explored by government. BMJ 2020; DOI: 10.1136/bmj.m1562.

12. Rimmer A. Covid-19: Two thirds of healthcare workers who have died were from ethnic minorities. BMJ 2020; DOI: $10.1136 /$ bmj.m1621.

13. UK Government. NHS workforce. 2020. Available online at https://www.ethnicity-facts-figures.service.gov. uk/workforce-and-business/workforce-diversity/nhsworkforce/latest (accessed May 2020).

14. British Dental Association. Live updates: Coronavirus and dentistry. 2020. Available online at https://bda.org/ advice/Coronavirus/Pages/latest-updates.aspx (accessed June 2020).

15. lacobucci G. Covid-19: NHS bosses told to assess risk to ethnic minority staff who may be at greater risk. $B M J$ 2020; DOI: 10.1136/bmj.m1820.

16. Welsh Government. COVID-19 workforce risk assessment tool. 2020. Available online at https://gov. wales/covid19workforceriskassessment-tool (accessed June 2020).

17. British Dental Association. Returning to work. 2020. Available online at https://bda.org/advice/Coronavirus/ Pages/returning-to-work.aspx (accessed June 2020).

18. Faculty of Occupational Medicine. COVID-19 Update: Risk Reduction Framework for NHS staff at risk of COVID-19 infection. 2020. Available online at https://www.fom. ac.uk/covid-19/updateriskreductionframework fornhsstaffatriskofcovid19infection (accessed June 2020).

19. Pulham E. Press Release. Available at https://www.resus. org.uk/media/statements/resuscitationcounciluk statementsoncovid19coronaviruscprandresuscitation covidhealthcareresources/pressreleasercukonlinetraining/ (accessed May 2020).

20. British Association of Oral \& Maxillofacial Surgeons. OMFS and COVID-19. 2020. Available at https://www. baoms.org.uk/professionals/omfs_and_COVID-19.aspx (accessed May 2020).

21. Office for National Statistics. Which occupations have the highest potential exposure to the coronavirus (COVID-19)? 2020. Available at https://www.ons. gov.uk/employmentandlabourmarket/peopleinwork/ employmentandemployeetypes/articles/which occupationshavethehighestpotentialexposuretothe coronaviruscovid19/2020-05-11 (accessed May 2020)

22. Faculty of General Dental Practice UK. FGDP(UK) \& CGDent launch UK guidelines for safe return to general dental practice during COVID-19. 2020. Available at https://www.fgdp.org.uk/news/fgdpukcgdent launchukguidelinessafereturngeneraldental practiceduringcovid19 (accessed June 2020).

23. Booth J. Dentists in Indonesia are dying from COVID-19. 2020. Available at https://coronavirus.dental-tribune. com/news/dentists-in-indonesia-are-dying-fromcovid-19/ (accessed May 2020).

24. Izzetti R, Nisi M, Gabriele M, Graziani F. COVID-19 Transmission in Dental Practice: Brief Review of Preventive Measures in Italy. J Dent Res 2020; DOI: 10.1177/0022034520920580.

25. Peng X, Xu X, Li Y, Cheng L, Zhou X, Ren B. Transmission routes of 2019-nCoV and controls in dental practice. Int J Oral Sci 2020; 12: 9.

26. Heneghan C, Brassey J, Jefferson T. COVID-19: What proportion are asymptomatic? 2020. Available at https:// www.cebm.net/covid-19/covid19whatproportionareasymptomatic/ (accessed June 2020). 